\title{
Recognition of online handwritten Gurmukhi characters based on zone and stroke identification
}

\author{
KARUN VERMA and R K SHARMA* \\ Department of Computer Science and Engineering, Thapar University, Patiala 47004, India \\ e-mail: karun.verma@thapar.edu; rksharma@thapar.edu
}

MS received 12 January 2015; revised 19 October 2016; accepted 25 October 2016

\begin{abstract}
Handwriting recognition is a technique that converts handwritten characters into a machine-processable format. Handwritten characters can either be presented to machine online or offline. A good amount of research in this area has been carried out for English, Chinese, Japanese and Korean languages. Research is also going on for Indian languages on developing online handwriting recognition systems. Headline and baseline are common features in most Indic languages which divide a character into three zones, namely, upper, middle and lower zones. Identification of headline and baseline is a major task for classification of strokes located in these three zones. A zone identification algorithm is proposed and tested in this text for online handwriting recognition of Gurmukhi script. The strokes are grouped into these separate zones and are recognized based on respective support vector machine model for each zone. A rule-based approach has also been applied and tested for generation of characters from the set of recognized strokes. In this work, an accuracy of $95.3 \%$ has been achieved for zone identification and an accuracy of $74.8 \%$ has been achieved for character identification for Gurmukhi script. This accuracy has been achieved when the recognition engines of three zones were tested on the dataset of 428 characters each written by 10 users.
\end{abstract}

Keywords. Gurmukhi script; SVM; handwriting recognition; pre-processing; post-processing.

\section{Introduction}

Computer human interface is one of the buzzwords these days owing to the invention of touch- and pen-based input devices in PDAs, and other computing devices. With the invention of these touch based interfaces, handwriting has become an important medium for interacting with the computers. In these devices, a sensor picks up the movements of pen or fingers between successive PEN UP/PEN DOWN events. This kind of data is known as digital ink and can be considered as a dynamic representation of handwriting, which can be directly converted to stream of characters of a language using online handwriting recognition system.

Online handwriting recognition system is the system by which a computer can recognize characters and other symbols written by hand in natural handwriting. A number of applications can be created to facilitate the processing of information that is mostly collected using forms to be filled by the people with these systems. A large amount of work in handwriting recognition has been done for simplified Chinese, traditional Chinese, English, Japanese, and Korean languages [1-4]. However, a little attention has been devoted to Indian scripts. Gurmukhi is the script used to

*For correspondence write Punjabi language in India. Graphemes (strokes) in Gurmukhi script can appear in three different zones, namely, upper, middle and lower zones as shown in figure 1 . The current work presents an approach to identify the zone in which the grapheme appears and then recognizes it with respective recognition engine giving zone wise accuracy. In this work, 7 stroke classes in upper zone, 80 stroke classes in middle zone, and 12 stroke classes in lower zone have been considered. The work also explores the postprocessing requirements of conversion of strokes into characters of Gurmukhi script.

This paper is organized into seven sections. This section introduces basic concepts behind the work. Section 2 describes the literature related to the problem. Section 3 describes Gurmukhi script and its features and how online handwritten data has been collected. This section also discusses various challenging issues in Gurmukhi script recognition and explains various stages of Gurmukhi character recognition system. Section 4 explains pre-processing steps applied on the collected data. This section also explains how various features have been calculated and then organized for recognition of strokes. Section 5 describes how classification models were chosen from the selected feature set. It also explains various parameters used for support vector machine to build the stroke classification model. Section 6 explains the zone identification 


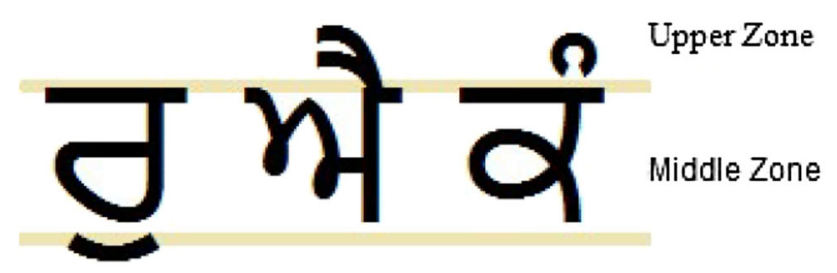

Lower Zone

Figure 1. Zone division of Gurmukhi characters.

algorithm that is used to identify the lower and upper baselines from a set of Gurmukhi strokes. This section also analyses the results of zone identification algorithm as well as the recognition of characters using rule based post-processing algorithm. Section 7 concludes the work done with salient findings.

\section{Related work}

This section briefly reviews the literature on online handwriting recognition systems. In the computing environment, keyboards had been an effective interface for interacting with computers, but with advancements in computational technology, and advent of touch-based systems, a more natural way of interacting with the computers is being explored. Tappert et al [1] reported a survey on online and offline handwriting recognition systems for Japanese, Korean, Chinese and English languages. Bellegarda et al [5] introduced the idea of recognizing handwriting using a computer-based method. The authors proposed a continuous parameter hidden Markov model-based approach to handwriting recognition systems. Dutta and Chaudhary [6] proposed a curvature feature-based recognition method for Bangla alphanumeric characters, and found the technique effective with two-stage feed-forward neural network. Garcia et al [7] presented a neural predictive system for online writer independent character recognition. A fixed number of predictive neural networks (NN) were used to model each letter by them. For 10 different writers, the system gave quite a good recognition rate and improvement in results was reported with the extension of the system to the durational HMM framework. Hu et al [8] proposed HMM-based algorithms for online handwritten character recognition. They achieved a writer independent recognition rate of $94.5 \%$ on 3,823 unconstrained online handwritten word samples from 18 writers covering a 32 word vocabulary. Takahashi et al [9] achieved a $90.0 \%$ recognition rate for 881 Kanji characters with fast HMM algorithm for handwriting recognition. Yaeger et al [10] presented number of innovations in application of artificial neural networks for classification of characters and word recognition. Plamondon and Sirhari [11] in their survey on online and offline handwriting recognition systems explained various processes involved starting from input to final understanding/recognition of characters for a language. They have discussed categories of recognition methods, namely, structural/rule based methods and statistical methods in their work in order to recognize handwriting with pen-based devices. Structural/rule based methods define robust and reliable rules for recognition purposes. In statistical methods, shape of a graphical mark known as stroke or grapheme is described by fixed number of features, and the classes of these graphical marks are described by multidimensional probability distribution. Connell et al [12] proposed a recognition system for unconstrained online Devanagari characters. An accuracy of $86.5 \%$ with no rejects was achieved by them. Jain et al [13] in their survey on pattern recognition cited statistical, syntactical and structural, neural network and elastic matching techniques as common handwriting recognition methods experimented by researchers. Connell and Jain [14] presented a template matching algorithm for online character recognition. The authors used these templates as reference for efficient classification of characters using decision trees. Pechwitz and Märgner [15] proposed a baseline detection algorithm to detect the orientation of a word. They explained the importance of baseline as a precondition to a handwriting recognition system. Skew normalization and character segmentation can be performed with the help of baseline in any script. Garain et al [16] presented promising results for online recognition of handwritten characters in Devanagari and Bangla, the two major Indian scripts, by modelling the human motor functionality while writing characters. Vijayasenan et al [17] applied principal component analysis on online handwriting recognition in Tamil script. Joshi et al [18] presented an automatic recognition system for isolated handwritten Devanagari characters by linearizing consonant conjuncts. Bharat and Madhvanath [19] proposed a data driven HMM based online handwriting recognition system for Tamil script. The authors reported encouraging results for exploring the approach for other Indic scripts. Jayaraman et al [20] used a baseline detection approach in their pursuit to recognition of Telugu script. They explained a modular approach to recognize Telugu script distributing the strokes into three classifiers based on upper and lower baselines in Telugu script. Parui et al [21] presented two- stage HMMbased method for recognition of Bangla characters. Sharma et al [22] explored the implementation of elastic matching for online handwritten Gurmukhi strokes and further formed the character based on recognized strokes. A character set of 41 Gurmukhi characters was tested by them for 60 writers and a recognition rate of $90.1 \%$ has been reported. Swethalakshmi [23] proposed handwriting recognition system for Devanagari and Tamil scripts using SVMs. She analyzed performance of different SVM classifiers and compared it with HMM based classifier using spatiotemporal representation of strokes. The stroke recognition accuracies of $95.3 \%$, and $92.1 \%$ have been reported in her work for Devanagari, and Tamil scripts, respectively. A character recognition accuracy of $89.9 \%$ for Devanagari script has also 
been reported in this work. Sundaram and Ramakrishnan [24] proposed Tamil script specific post-processing schemes for improving the recognition rate of online handwritten characters in Tamil using nearest neighbor classifier. Sharma [25] proposed a post-processing algorithm for rearrangement of strokes to identify Gurmukhi characters from combination of strokes. The authors achieved a character level recognition accuracy of $81.0 \%$ for a set of 2,576 Gurmukhi words. Prasad et al [26] presented a divide and conquer technique based approach to recognize Kannada characters by dividing the strokes into various auxiliaries and using classifiers for each of the auxiliary to predict the Kannada character using a rule base. Sharma et al [27-29] worked on 49 classes for online handwritten character recognition of Gurmukhi script. They did not consider zoning in their work as the number of classes considered by them was limited. Hajj and Awad [30] presented an efficient handwriting recognition system for writer independent isolated letter recognition for English. They modelled pen trajectories for feature extraction and applied multi-stage support vector machines (SVMs) for classification of strokes based on zone. Sharma et al [31] presented a study of SVM based classifiers to develop online handwriting recognition system for Gurmukhi script. Kumar and Sharma [32] proposed a post-processing algorithm for generation of Gurmukhi characters from a set of intermediate strokes created by recognition system. In the current work, we have presented a Gurmukhi character recognition system. This system first identifies the zone of a stroke and then recognizes it. The recognized strokes are used to form the Gurmukhi character guided by a rule base. Motivated by speech recognition framework, Kopparapu and Lajish [33] proposed a novel framework for online handwritten Devanagari character recognition. A feature set called fuzzy directional features (FDF) has been introduced by them. Three classifiers, namely, nearest neighbor classifier, dynamic time warping (DTW), and SVM, have been used by the authors to test the system. They reported a character level accuracy of 73.9\% using FDF with SVM classifier. Table 1 illustrates some of the salient works done for developing online character recognition systems for Indic languages.

\section{Gurmukhi script and data collection}

In this work, we have addressed the problem of classifying different characters of Gurmukhi script in three zones and then classifying the strokes in each zone. Gurmukhi script is one of the popular scripts in north India. This is also popular in Punjab province of Pakistan. Besides, this script is very frequently used by Punjabis settled in Canada, USA, UK, Australia and other countries. Table 2 contains the characters used in Gurmukhi script. Writing style in this script is from left to right. Gurmukhi script contains 41 consonants as given in table 2(a). Out of these 41 consonants, there are 35 base consonants or akhars (A0-G4) and 6 consonants with subscript dot (A5-F5). В, भ, ᄅ are the three vowel holders in Gurmukhi script. A vowel holder is used when there is no consonant between vowel sounds. This script contains 10 vowel modifiers or mātrās, 9 of them as given in table 2 (b) have specified symbols (A7-I7). These are also known as dependent vowels and remaining one has no symbol and is known as mukta. A mukta is pronounced between each and every consonant wherever no other vowel is present unless otherwise indicated. Table 2(b) also exemplifies the usage of these dependent vowels with a consonant $\alpha$ (A8-I8). The first column in table 2(b) (A6-I6) shows independent vowels

Table 1. Work done for Indic languages.

\begin{tabular}{|c|c|c|c|}
\hline Language & References & Classifier & Dataset and recognition accuracy \\
\hline \multirow[t]{2}{*}{ Bangla } & Dutta and Chaudhury [6] & Feed forward neural network & $\begin{array}{c}90.0 \% \text { for Bangla numerals and } 85.0 \% \text { for Bangla } \\
\text { characters }\end{array}$ \\
\hline & Parui et al [21] & HMM & $\begin{array}{c}24,500 \text { online handwritten isolated characters, } \\
84.6 \% \text { stroke level recognition accuracy }\end{array}$ \\
\hline \multirow[t]{4}{*}{ Hindi } & Connell et al [12] & $\begin{array}{c}\text { HMM and nearest neighbor stroke } \\
\text { recognizer }\end{array}$ & $\begin{array}{l}20 \text { writers wrote five samples of each character, } \\
86.5 \% \text { character level recognition accuracy } \\
\text { with no rejects }\end{array}$ \\
\hline & Joshi et al [18] & Subspace method for classification & $94.5 \%$ accuracy for Devanagari characters \\
\hline & Swethalakshmi [23] & Support vector machines & $\begin{array}{l}89.9 \% \text { character recognition accuracy on a } \\
\text { dataset of } 3,774 \text { words from } 34 \text { writers }\end{array}$ \\
\hline & $\begin{array}{l}\text { Bharath and Madhvanath } \\
\text { [19] }\end{array}$ & $\begin{array}{c}\text { Lexicon driven HMM and lexicon free } \\
\text { HMM }\end{array}$ & $\begin{array}{c}87.1 \% \text { recognition accuracy on } 20,000 \text { word } \\
\text { lexicon when the two recognizers are } \\
\text { combined }\end{array}$ \\
\hline Kannada & Prasad et al [26] & Rule-based classifier & $\begin{array}{l}81.0 \% \text { character recognition accuracy with } \\
17,181 \text { character samples }\end{array}$ \\
\hline Punjabi & Sharma et al [22, 27-29] & Elastic matching, HMM & $\begin{array}{c}\text { 91.9\% character recognition accuracy with } 40 \\
\text { stroke classes }\end{array}$ \\
\hline Tamil & $\begin{array}{c}\text { Bharath and Madhvanath } \\
\text { [19] }\end{array}$ & Lexicon driven HMM, & $\begin{array}{l}91.8 \% \text { recognition accuracy with } 20,000 \text { word } \\
\text { lexicon } u\end{array}$ \\
\hline Telugu & Jayaraman et al [20] & SVM based classifier & $83.0 \%$ character recognition accuracy \\
\hline
\end{tabular}


Table 2. Chart of Gurmukhi characters.

\begin{tabular}{|c|c|c|c|c|c|c|}
\hline & 0 & 1 & 2 & 3 & 4 & 5 \\
\hline \multicolumn{7}{|c|}{ (a) consonants in Gurmukhi script } \\
\hline $\mathrm{A}$ & ७ & म & Z & म & J & म \\
\hline $\mathrm{B}$ & र & \& & गा & щ & ฐ & ?⿻्भ \\
\hline $\mathrm{C}$ & $\exists$ & ह & त & ₹ & ह & गा \\
\hline $\mathrm{D}$ & ट & б & z & ह & ¿ & กุ \\
\hline $\mathrm{E}$ & 3 & घ & 已 & य & চ & .ढ \\
\hline $\mathrm{F}$ & य & б & घ & उ & $H$ & ড় \\
\hline \multirow[t]{2}{*}{$\mathrm{G}$} & ज & ஏ & ম & ह & इ & \\
\hline & & & & & & \\
\hline
\end{tabular}

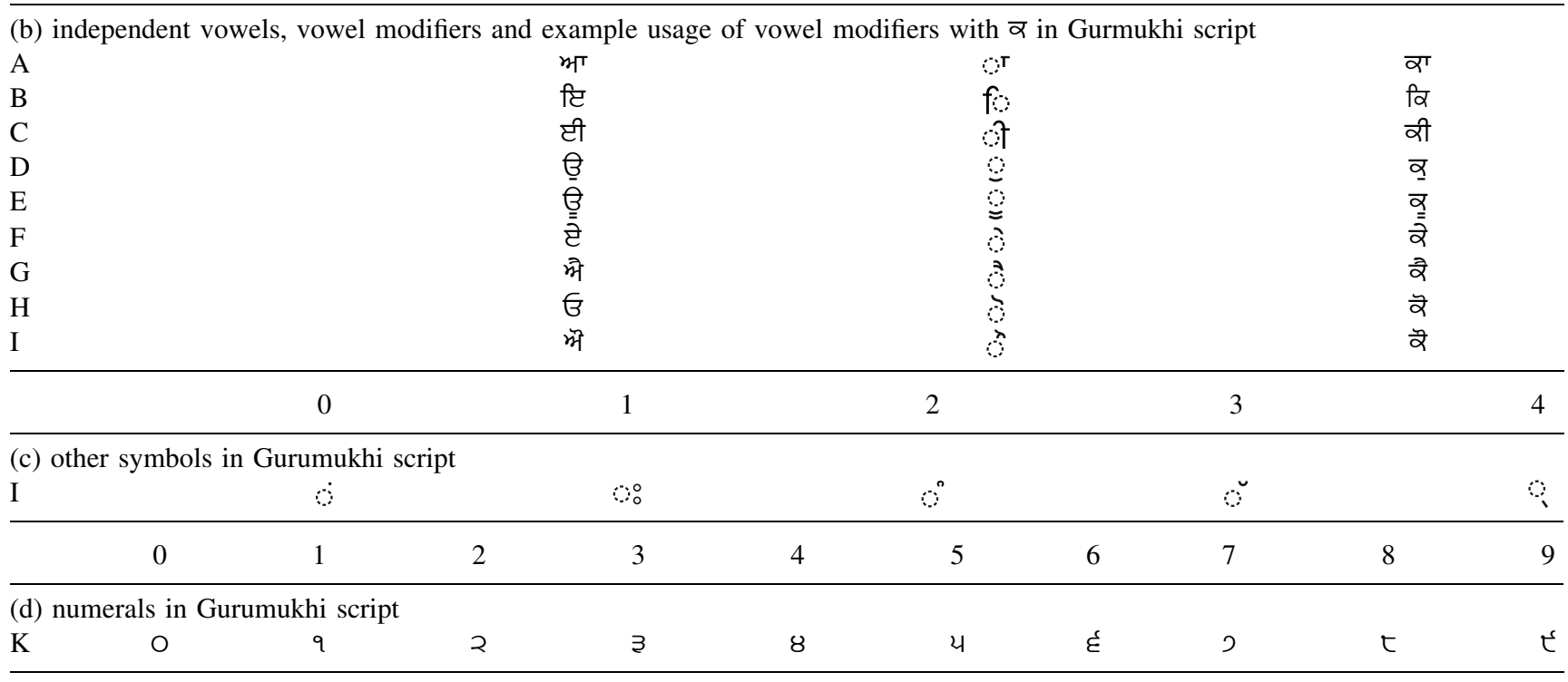

used in Gurmukhi script. The vowel symbols can appear above, below, or to either side of consonants, or their respective vowel holders. There are some other symbols used in Gurmukhi script to represent nasal, duplicity and other sounds, as shown in table 2(c). The script has two symbols for nasal sounds bind $\bar{\imath}$ (I0) and tippi (I2), and one symbol which duplicates the sound of any consonant, namely, addak (I3). The two remaining symbols halant (I4) and visarg (I1) are rarely used in modern Gurmukhi texts, and have been used occasionally in Sanskritised text or in dictionaries for extra phonetic information. Table 2(d) represents numeral digits written in Gurmukhi script. Seven Gurmukhi characters (, , J, ઇ, ट, उ, ज, ह) may also appear in the lower zone and are known as half characters (paireencharacters). These half characters have not been allocated any Unicode, and are dealt with functions using halant.

\subsection{Some issues in Gurmukhi script recognition}

In section 2, we surveyed online handwritten word/character recognition systems for some languages. The major issues tackled by authors in developing these systems include shape complexity of characters, presence and absence of baseline, features for recognition of characters, and stroke/character level recognition. Following subsection explains some of the issues that play an important role in developing an online handwritten recognition system for Gurmukhi script.

3.1a Three zones of writing strokes: Gurmukhi characters are written in various styles that increase the difficulty in recognition of a particular character. Gurmukhi script characters may lie in three horizontal zones, namely, upper zone, middle zone and lower zone as depicted in figure 1. The upper zone denotes the region above the headline, where some of the vowels $(\lambda, \vec{y})$ and sub-parts of some other vowels (f, it) reside, while the middle zone represents the area below the headline where the consonants and sub-parts of some vowels (f, it) are present. Middle zone consists of most of the Gurmukhi characters. The lower zone represents the area below middle zone where some vowels and certain half characters lie in the foot of consonants. A Gurmukhi character can be written with single or a combination of strokes, with stroke considered as smallest unit. These strokes may appear in a single zone, or combination of three zones described above. To initiate the recognition process, various classes of strokes have been identified. These stroke classes are 
further divided into three zones, namely, upper, middle and lower zones to avoid confusion between similar looking strokes in three zones.

3.1b Variations in writing style: The personal characteristics in handwriting variations include writer's choice of strokes for writing same character. Different users write characters with different choices of strokes and their sequence. For example, in order to write the character $₹$, several combinations of strokes can be used as illustrated in figure 2, where each stroke is written without knowing the zone boundaries. Since all the strokes have been grouped according to the zone in which they appear, it is difficult to figure out zone boundaries by looking at the stroke. The sequence of strokes may also vary for writing a character leading to confusion at post-processing level as illustrated in figure 3 for character 8 . While writing in Gurmukhi, the writer adds an upper bar on characters to end the word. A writer has also to add a middle bar in some characters that is similar in shape to the upper bar. Presence or absence of upper and middle bar with other strokes generates a different character in Gurmukhi. Some example combinations are shown in figure 4. 3.1c Presence of half characters: In addition to the problems mentioned above, another challenge is to handle strokes in lower zone that are half characters like paireen

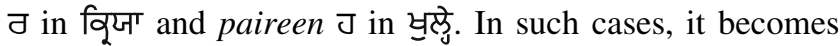
sometimes difficult to draw zone boundaries between the middle and lower zones, leading to difficulties in recognition of these half characters. These half characters may then conflict with other characters like ₹, চ, こ, চ.

\subsection{Steps involved in recognition of characters}

In order to recognize online handwritten characters, the set of strokes goes through the phases as illustrated in figure 5 . This section includes a brief description of these phases:

(a) An online handwritten stroke is written by a user on tablet using a touch device or pen device. Each stroke consists of $x-y$ trace points between successive PEN DOWN and PEN UP movements.

(b) These strokes are then fed to stroke zoning phase which identifies the zone of the strokes, based on the relative position of strokes with respect to other strokes. The three zones in which a stroke may lie can be upper, middle or lower zone as illustrated in previous

\begin{tabular}{|c|c|c|c|c|}
\hline \multirow[t]{2}{*}{ Combination } & \multicolumn{3}{|c|}{ Stroke(s) (zone) } & \multirow{2}{*}{ Outcome } \\
\hline & 1 & 2 & 3 & \\
\hline 1 & $\frac{1}{2}$ (Middle) & & & ₹ \\
\hline 2 & $\breve{2}$ (Middle) & - (Upper) & & ₹ \\
\hline 3 & y (Middle) & $\alpha$ (Middle) & & \$ \\
\hline 4 & y (Middle) & $\alpha$ (Middle) & - (Upper) & ₹ \\
\hline
\end{tabular}

Figure 2. Example combinations of strokes for writing ₹.

\begin{tabular}{|c|c|c|c|c|}
\hline \multirow{2}{*}{ Combination } & \multicolumn{3}{|c|}{ Stroke sequence (zone) } & \multirow{2}{*}{ Outcome } \\
\hline & 1 & 2 & 3 & \\
\hline 1 & $\varepsilon$ ( Middle) & 7 ( Middle) & & घ \\
\hline 2 & I (Middle) & $\varepsilon$ ( Middle) & & $\begin{array}{l}\text { ए, ह (confused } \\
\text { with घ) }\end{array}$ \\
\hline 3 & q ( Middle) & - ( Upper) & & घ \\
\hline 4 & $\varepsilon$ (Middle) & ( Middle) & - ( Upper) & घ \\
\hline
\end{tabular}

Figure 3. Example of sequence of strokes for writing घ. 


\begin{tabular}{|c|c|c|c|c|}
\hline \multirow{2}{*}{ Combination } & \multicolumn{3}{|c|}{ Stroke number (zone) } & \multirow{2}{*}{$\begin{array}{l}\text { Required } \\
\text { outcome }\end{array}$} \\
\hline & 1 & 2 & 3 & \\
\hline 1 & u (Middle) & & & ૫ \\
\hline 2 & ż ( Middle) & & & ધ \\
\hline 3 & U (Middle) & - ( Middle) & & Y \\
\hline 4 & z (Middle) & - ( Upper) & & घ \\
\hline 5 & 2 ( Middle) & I ( Middle) & & ૫ \\
\hline 6 & य (Middle) & - ( Middle) & - ( Upper) & घ \\
\hline 7 & 2 ( Middle) & 7 ( Middle) & - ( Middle) & घ \\
\hline 8 & 2 ( Middle) & I ( Middle) & - ( Upper) & य \\
\hline 9 & 2 ( Middle) & I ( Middle) & - ( Middle) & 证 \\
\hline
\end{tabular}

Figure 4. Examples of common ligatures for घ, Ұ, य, य.

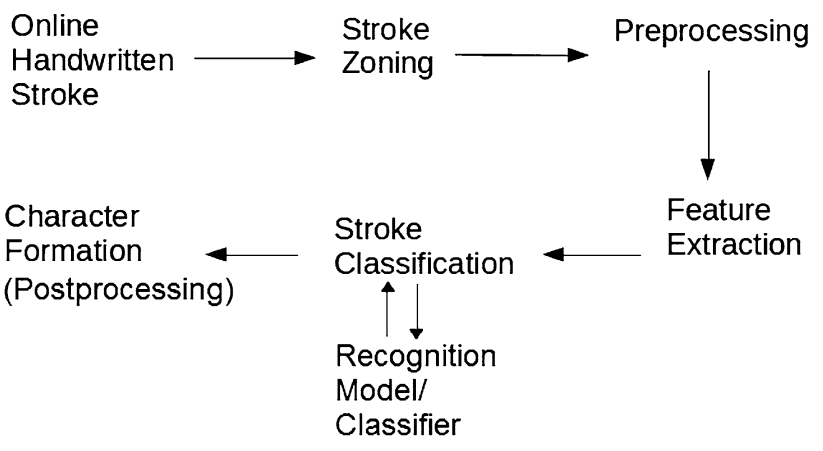

Figure 5. Steps in character recognition.

subsection. The actual $x-y$ points of the stroke are used in further processing.

(c) After identifying the zone, the stroke points are preprocessed for extraction of feature in this step. During pre-processing, a noise or distortion that arises due to software limitations is removed from original stroke. Now, normalization and centering of stroke is done, where the input stroke is fitted into a fixed size window, and is moved to the central location. During writing of a stroke, some points might be missed by the touch sensor. These missing points are interpolated using Bézier interpolation technique. Jitters are removed from the stroke by averaging each point with its neighbors. (d) Features required for classification of strokes, as per model chosen, are now extracted from the strokes which have further been used for recognition of the strokes.

(e) Support vector machine is next used for classification of strokes. The recognition accuracy using linear, polynomial, radial basis and sigmoid kernels are then calculated for different combinations of parameters.

(f) After classification of strokes, post-processing steps are applied on the recognized strokes to identify the character formed using these strokes.

\subsection{Data collection}

Samples of Gurmukhi words have been collected using Dell Latitude XT3 Tablet PC. Individual strokes with their sequence have been recorded in XML format. An $800 \times$ 800 interface for writing strokes is used. Each stroke consists of $x-y$ traces of digital PEN on the touch screen between successive PEN DOWN and PEN UP movements. In the data collection phase, 44,301 samples of 2,000 unique Gurmukhi words containing 241,239 strokes have been collected from 124 different writers. The writers are chosen from various parts of Punjab, who are familiar with writing Gurmukhi script and are using Gurmukhi script in their day-to-day life. Among the users, there are school children studying in class 6 and above up to the degree/ diploma level, Government officials at local offices in 
Table 3. Upper zone strokes and their associated strokeIDs.

\begin{tabular}{|c|c|c|c|c|c|c|c|}
\hline stroke & 1 & $\checkmark$ & 4 & 1 & $\smile$ & . & 0 \\
\hline strokeID & 122 & 123 & 124 & 125 & 126 & 127 & 128 \\
\hline stroke & 1 & ? & 9 & $\pi$ & $b$ & & \\
\hline strokeID & 130 & 131 & 132 & 133 & 134 & & \\
\hline
\end{tabular}

Punjab, research staff involved in development of Gurmukhi script and some commoners who have studied Punjabi at their school level. Based on the writing styles of users and stroke formation, the strokes' data is classified into two different classes, namely, Class $A$ and Class $B$. Class A data consists of those strokes that have been written by users as per standard way of writing script in an unconstrained environment. While Class $B$ data consists of those strokes that do not meet standards of script writing and are also written in an unconstrained environment. This is worth mentioning here that one needs to prepare a different set of rules for Class A and Class B data. Out of these strokes, 99 stroke classes that have a higher frequency of occurrence in Class A and Class B data have been identified and are further used in this work. The data collected from these users have been annotated at the stroke level with unique StrokeIDs associated with each stroke. Tables 3, 4 and 5 contain the strokes and the strokeIDs associated with them in three zones.

Table 4. Middle zone strokes and their associated strokeIDs.

\begin{tabular}{|c|c|c|c|c|c|c|c|c|c|c|}
\hline stroke & - & G & 8 & 6 & $m$ & $r$ & c & ' & u & c \\
\hline strokeID & 121 & 141 & 142 & 143 & 144 & 145 & 146 & 147 & 148 & 149 \\
\hline stroke & Y & j & d- & म & 7 & $d$ & J & $\alpha$ & 2 & 4 \\
\hline strokeID & 150 & 151 & 152 & 153 & 154 & 155 & 156 & 157 & 158 & 159 \\
\hline stroke & 바 & 2 & 1 & - & $d$ & $\partial$ & $2 y$ & $w$ & 夕 & 5 \\
\hline strokeID & 160 & 161 & 162 & 163 & 164 & 165 & 166 & 167 & 168 & 169 \\
\hline stroke & of & 8 & $\delta$ & है & q & 9 & $\breve{2}$ & $y$ & $\varepsilon$ & $\varepsilon$ \\
\hline strokeID & 170 & 171 & 172 & 173 & 174 & 175 & 176 & 177 & 179 & 180 \\
\hline stroke & $\varepsilon$ & c & d & 0 & 3 & 5 & \& & ? & $c$ & 3 \\
\hline strokeID & 181 & 182 & 183 & 184 & 185 & 186 & 187 & 188 & 190 & 191 \\
\hline stroke & 3 & $\tau$ & z & 7 & $\pi$ & ) & $n$ & 6 & $\bar{\epsilon}$ & $\varepsilon$ \\
\hline strokeID & 192 & 193 & 194 & 195 & 196 & 197 & 198 & 200 & 201 & 202 \\
\hline stroke & $q$ & o & 3 & म & v & F & अ & $x$ & v & 3 \\
\hline strokeID & 203 & 204 & 205 & 206 & 207 & 208 & 209 & 210 & 211 & 212 \\
\hline stroke & 3 & $\rho$ & q & j & 1 & $\overline{9}$ & $q$ & 4 & $\zeta$ & $\varepsilon$ \\
\hline strokeID & 214 & 215 & 216 & 217 & 218 & 222 & 223 & 224 & 225 & 226 \\
\hline
\end{tabular}

Table 5. Lower zone strokes and their associated strokeIDs.

\begin{tabular}{|c|c|c|c|c|c|c|c|}
\hline stroke & $\mathcal{V}$ & & $\ddots$ & $\jmath$ & $\varepsilon$ & $\boldsymbol{z}$ & v \\
\hline strokeID & 101 & 102 & 103 & 104 & 105 & 106 & 107 \\
\hline
\end{tabular}

\section{Pre-processing and feature extraction}

For developing the recognition model for classification of strokes, 100 samples of each stroke from three zones have been selected from the database. The strokes are selected in equal proportion from all the users, so as to reflect all patterns for a single stroke. During pre-processing, a series of transformations is performed on the stroke points. These transformations are normalization, centering, interpolation and smoothing as explained in previous section. The standard algorithms as explained by Guerfali and Plamondon [34] and Trier et al [35] have been used to implement these transformations. After performing the pre-processing steps, we obtained a $500 \times 500$ window containing resampled 64 $x-y$ points. These resampled 64 points are normalized features that have been considered for building SVM based classification models.

\section{Zone identification}

Identification of the zone of a stroke is an important process in the recognition model proposed in this work. Identification of the zone is critical for further classifying the stroke. We have proposed an algorithm MarkStrokeZone in this work that identifies the zone of a given stroke. The original $x-y$ points of each stroke are utilized in this algorithm to identify the boundaries of middle zone: $M i d Y_{\min }$ and $M i d Y_{\max }$. We have considered top left corner of data capturing device as the origin in this work. As such, $M i d Y_{\min }$ is upper and $M i d Y_{\max }$ is lower boundary of middle zone. Center is average of MidY $Y_{\min }$ and $M i d Y_{\max }$ denoting the middle value of $y$-coordinate in middle zone. These boundaries once created are used to mark the zone of the stroke. As per statistics collected from database, first stroke mostly occurs in middle zone, so in the proposed algorithm it has been assumed that the first stroke is always written in middle zone. After identifying the middle zone boundaries of the stroke, zone identification is done for next sequence of strokes. Here, Stroke $Y_{\min }$ is the smallest $y$-coordinate and Stroke $Y_{\max }$ is the largest $y$-coordinate of new stroke. Center $_{n e w}$ is the average $y$-coordinate value of new stroke and denotes the center of new stroke. If Stroke $Y_{\max }$ of new stroke is less than $\operatorname{Mid}_{\min }+h$ (a pre-defined tolerance level calculated based on statistics of collected data), then stroke considered to lie in upper zone. Similarly, if new stroke's Stroke $Y_{\min }$ is greater than middle zone's $M i d Y_{\min }-h$, then the stroke is considered to lie in lower zone. If the $S_{t r o k e} Y_{\min }$ of the new stroke is greater than Center and StrokeY $Y_{\max }$ is less than $M i d Y_{\max }+h$, then the stroke is considered in lower zone. For all other cases, the new stroke is considered to lie in middle zone. The strokes are sent to respective recognition engines for identification based on their zone. All the cases described above are illustrated in figure 6. 


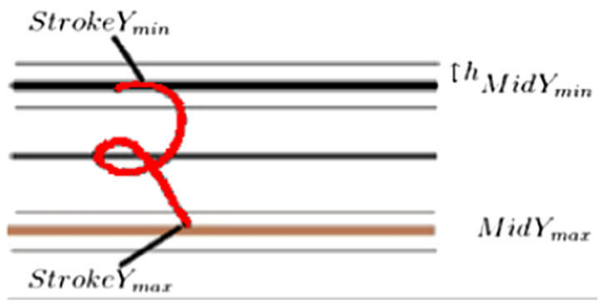

(a)

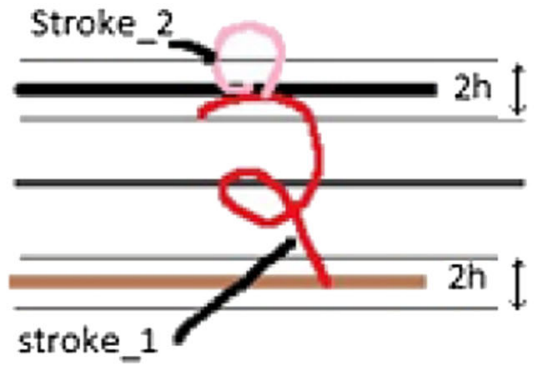

(c)

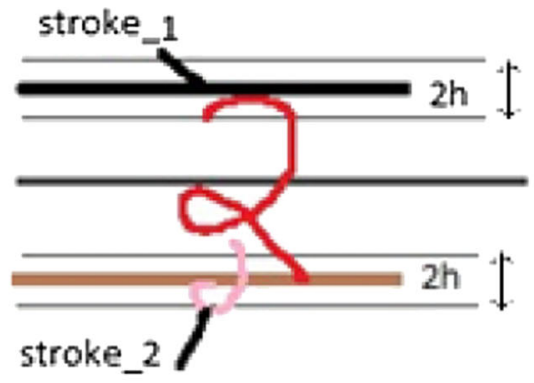

(e)

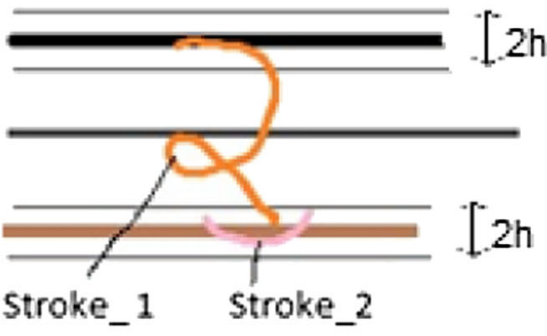

(b)

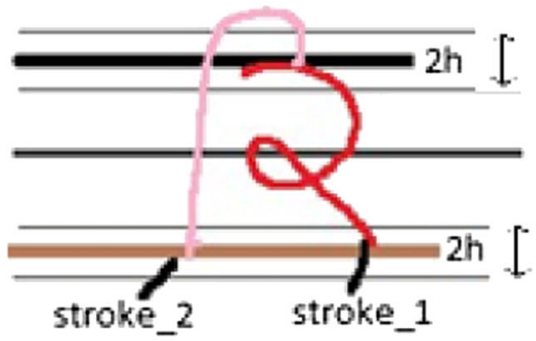

(d)

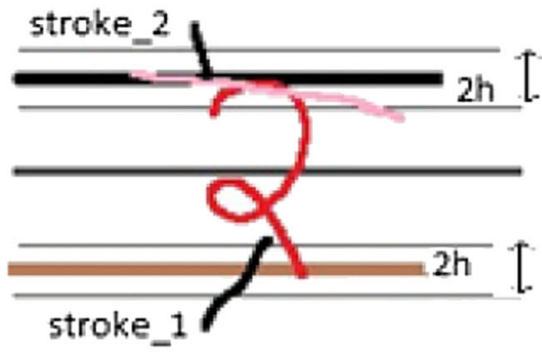

(f)

Figure 6. Cases of zone identification: (a) initialization of middle zone boundaries, (b) identification of lower zone stroke, (c) identification of upper zone stroke, $(\mathbf{d})$ identification of middle zone stroke, (e) case for paireen alphabets, and (f) special case for handling upper bar.

\subsection{Verification and validation of zone identification}

The values of parameters used in zone identification algorithm MarkStrokeZone have been inferred by analyzing the data collected from all users involved in data collection phase. This algorithm has been verified on a dataset of 4,500 characters used in training phase. These characters consisted of 4,870 strokes in upper zone, 8,340 strokes in middle zone and 2,873 strokes in lower zone. In this verification phase, an accuracy of $95.6 \%, 99.5 \%$ and $96.2 \%$ has respectively been achieved for upper, middle and lower zones.

For validation of the proposed algorithm for zone identification, a set of 4,280 handwritten Gurmukhi characters has been collected from 10 new users, who have not participated in the data collection activity for preparation of recognition models. This dataset consists of 15,297 strokes. Out of these 15,297 strokes; 4,632 strokes were in upper zone, 7,832 strokes were in middle zone and 2,733 strokes were in lower zone. Handwritten characters collected from these 10 users have been tested for zone identification and also for stroke recognition. Accuracy for zone identification is verified by manually visualizing the actual zone of stroke and the zone predicted by the zone identification algorithm. Table 6 depicts the accuracy of zone prediction for various strokes in upper, middle, and lower zones. Zone accuracy is defined as the number of strokes correctly marked in a zone upon total number of strokes marked for the zone. Average accuracy achieved for zone identification of strokes in upper, middle and lower zones attained in this work is $94.6 \%, 98.4 \%$, and $92.8 \%$, respectively. 


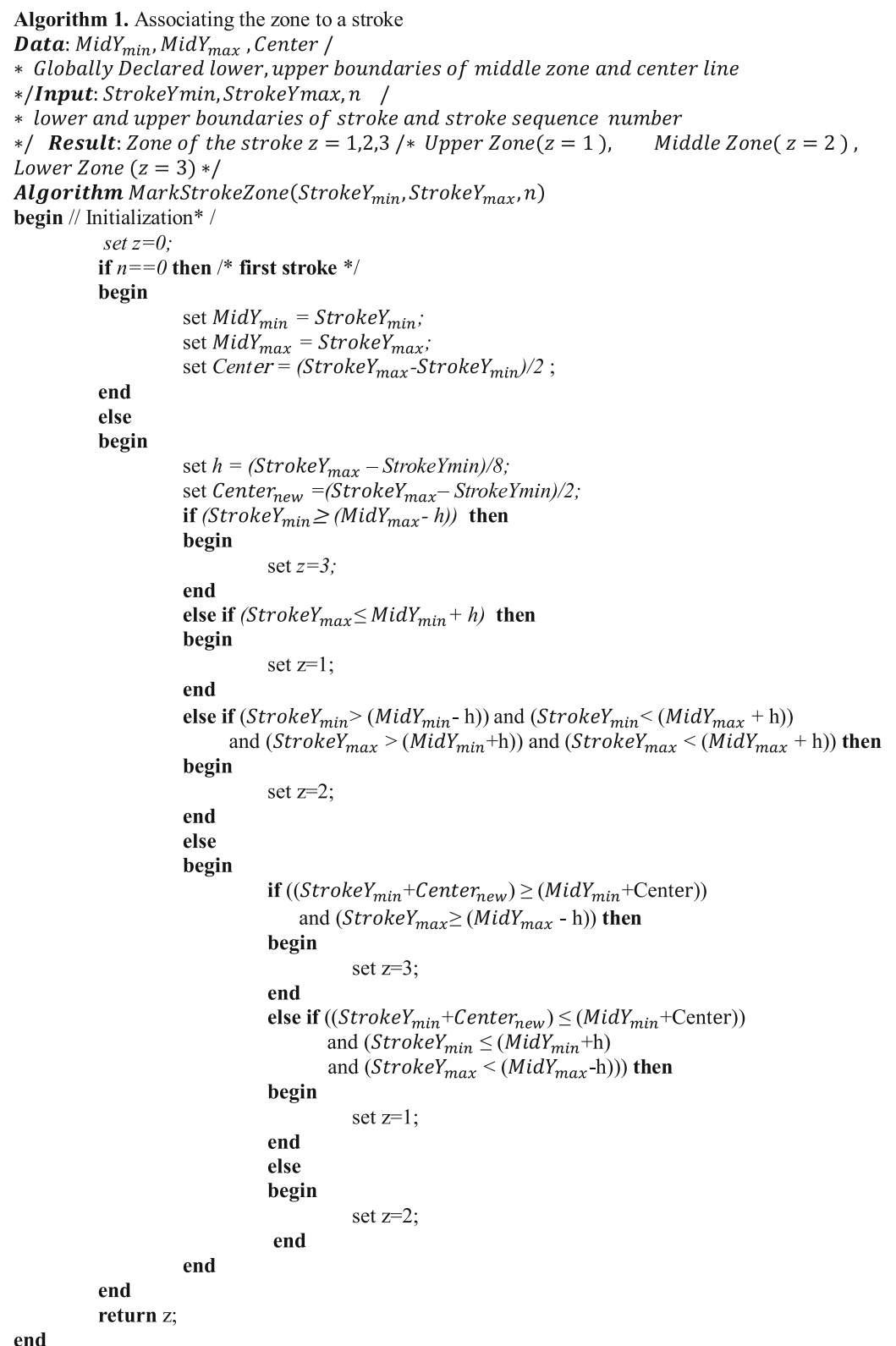

\section{Classification models}

As mentioned earlier, SVM recognition models are used for classification of strokes after identification of zone for given stroke. As such, three classification engines/models for upper, middle and lower zones have been developed by selecting one hundred samples of each stroke. There are 1,200 samples in upper zone, 8,000 samples in middle zone, and 700 samples in lower zone that have been used to train the recognition engines. In this work, as mentioned in previous section, normalized $x-y$ points have been used as feature. For the classification process, SVM with kernels, namely, linear, polynomial, sigmoid, and RBF have been used in this work. Other parameters that have empirically been experimented in this work are learning rate $(\gamma)$, penalty parameter $(C)$ and tolerance limit of termination $(\epsilon)$. Recognition rates for different experiments have been obtained using $k$-fold cross validation for three values of $k$ as mentioned in table 7. Table 7 also contains the values of other parameters considered in this work.

This is worth mentioning here that we have experimented 1,080 different SVMs based on these parameters in this work. Zone-wise $k$-fold cross validation optimal accuracy for the parameters considered in this work is given in table 8. 
Table 6. Userwise zone identification accuracy.

\begin{tabular}{lcccr}
\hline User & Upper zone $(\%)$ & Middle zone $(\%)$ & Lower zone $(\%)$ & Overall zone identification $(\%)$ \\
\hline User 1 & 91.4 & 98.6 & 94.8 & 94.9 \\
User 2 & 96.7 & 99.6 & 97.7 & 98.0 \\
User 3 & 94.4 & 99.0 & 94.3 & 95.9 \\
User 4 & 95.3 & 99.8 & 94.7 & 96.6 \\
User 5 & 98.1 & 97.8 & 81.5 & 92.5 \\
User 6 & 92.3 & 96.6 & 98.0 & 95.6 \\
User 7 & 95.3 & 97.3 & 85.8 & 92.8 \\
User 8 & 94.9 & 99.5 & 96.0 \\
User 9 & 91.7 & 97.5 & 93.5 & 93.9 \\
User 10 & 95.9 & 98.6 & 92.5 & 96.4 \\
Average & 94.6 & 98.4 & 94.7 & 95.3 \\
\hline
\end{tabular}

Table 7. SVM parameters.

\begin{tabular}{|c|c|}
\hline Parameter & Options/values considered \\
\hline Number of folds $(k)$ & $4,5,6$ \\
\hline Kernel & $\begin{array}{c}\text { Linear (0), Polynomial (1), RBF (2) } \\
\text { and Sigmoid (3) }\end{array}$ \\
\hline Learning rate $\left[\log _{2}(\gamma)\right]$ & -15 (2) 3 \\
\hline $\begin{array}{l}\text { Penalty parameter } \\
\quad\left[\log _{2}(C)\right]\end{array}$ & $-5(2) 15$ \\
\hline $\begin{array}{l}\text { Tolerance limit of } \\
\text { termination }(\epsilon)\end{array}$ & 0.001 \\
\hline
\end{tabular}

\subsection{Stroke recognition}

Stroke recognition accuracy is defined as the total number of strokes correctly classified over total number of strokes correctly marked for the zone. The stroke recognition accuracy for 10 users is illustrated in table 9. The average stroke recognition accuracies for upper, middle and lower zones are 96.3\%, 94.6\%, and 90.2\%, respectively.

\subsection{Character recognition}

To predict a character from the set of strokes that appear after the recognition process, a rule-based approach has been developed. These rules define the combination of strokes to create a character. These rules have been developed by analyzing the collected dataset for various combinations of strokes in which character can be written. A post-processing algorithm proposed by Kumar and Sharma [32] has been used to predict the character after the recognition of stroke. During the testing of recognition process, the test set consisted of 41 Gurmukhi characters, along with their combinations with two nasal symbols $(\dot{i}, \overrightarrow{0})$ addak $(\vdots)$, and nine vowel modifiers

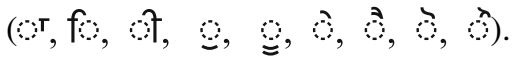

Character recognition accuracy of each user is shown in table 10, where it can be seen that for a character without mātrā and nasal symbols, the accuracy is in the range of 91.0-94.0\% with an average of $92.9 \%$. While recognizing complex character combinations with nasal, mātrās and both, the average accuracy goes down to $85.0 \%, 69.2 \%$ and $52.9 \%$, respectively. The major reason behind decrease of recognition rates is the variations in sequence of strokes for writing these complex characters in Gurmukhi script. Overall character recognition accuracy achieved in this work is $74.8 \%$.

\section{Results and discussion}

In this paper, a handwritten character recognition system for Gurmukhi script has been proposed. A zone identification algorithm for classification of strokes written in upper, middle and lower zones for Gurmukhi script has also been proposed, verified and tested. Recognition of a stroke has been done using SVM with a feature set of normalized $x-y$ points of the stroke. Zone identification algorithm performs well with an overall accuracy of $95.3 \%$. In an earlier experiment, [27] experimented with 40 classes on Gurmukhi handwritten character recognition system and

Table 8. Zone-wise optimum performance of SVM kernels.

\begin{tabular}{lccc}
\hline Kernel & $\begin{array}{c}\text { Lower zone performance }(\%) \text { at } \\
\left(k ; \log _{2}(\gamma) ; \log _{2}(C)\right)\end{array}$ & $\begin{array}{c}\text { Middle zone performance }(\%) \text { at } \\
\left(k ; \log _{2}(\gamma) ; \log _{2}(C)\right)\end{array}$ & $\begin{array}{c}\text { Upper zone performance }(\%) \text { at } \\
\left(k ; \log _{2}(\gamma) ; \log _{2}(C)\right)\end{array}$ \\
\hline Linear & $94.7(4 ;-7 ;-5)$ & $92.3(5 ;-7 ;-1)$ & $88.1(5 ;-7 ;-1)$ \\
Polynomial & $94.7(4 ;-1 ; 15)$ & $93.0(4 ;-7 ;-1)$ & $91.8(5 ;-13 ; 11)$ \\
RBF & $89.3(5 ;-15 ; 1)$ & $86.5(4 ;-15 ; 1)$ & $86.3(5 ;-15 ; 1)$ \\
Sigmoid & $14.9(3 ;-15 ; 5)$ & $2.4(4 ;-11 ; 15)$ & $17.2(4 ;-11 ; 3)$ \\
\hline
\end{tabular}


Table 9. Userwise stroke recognition accuracy.

\begin{tabular}{lcccc}
\hline User & Upper zone $(\%)$ & Middle zone $(\%)$ & Lower zone $(\%)$ & Overall stroke recognition accuracy $(\%)$ \\
\hline User 1 & 95.7 & 91.5 & 85.1 & 90.8 \\
User 2 & 97.0 & 97.2 & 94.1 & 96.1 \\
User 3 & 97.2 & 97.4 & 90.8 & 95.1 \\
User 4 & 95.3 & 93.0 & 90.8 & 93.0 \\
User 5 & 97.4 & 93.6 & 93.6 & 94.9 \\
User 6 & 95.3 & 95.2 & 86.0 & 92.2 \\
User 7 & 96.9 & 93.8 & 92.1 & 94.2 \\
User 8 & 96.6 & 95.8 & 89.5 & 93.9 \\
User 9 & 95.4 & 95.2 & 88.5 & 93.0 \\
User 10 & 96.1 & 93.7 & 91.9 & 93.9 \\
Average & 96.3 & 94.6 & 90.2 & 93.7 \\
\hline
\end{tabular}

Table 10. Userwise character recognition accuracy.

\begin{tabular}{lccccc}
\hline User & $\begin{array}{c}\text { Character without } \\
\text { mātrās }(\%)\end{array}$ & $\begin{array}{c}\text { Character with } \\
\text { mātrās }(\%)\end{array}$ & $\begin{array}{c}\text { Character with nasals } \\
\text { and addak }(\%)\end{array}$ & $\begin{array}{c}\text { Character with nasals, addak } \\
\text { and mātrās }(\%)\end{array}$ & $\begin{array}{c}\text { Average character } \\
\text { recognition }(\%)\end{array}$ \\
\hline User 1 & 91.7 & 51.5 & 100.0 & 50.0 & 73.3 \\
User 2 & 92.1 & 79.8 & 100.0 & 55.4 & 81.8 \\
User 3 & 93.2 & 77.1 & 50.0 & 52.7 & 68.2 \\
User 4 & 90.2 & 67.6 & 50.0 & 53.7 & 65.4 \\
User 5 & 90.6 & 65.3 & 100.0 & 52.7 & 77.2 \\
User 6 & 92.0 & 73.3 & 100.0 & 55.3 & 80.2 \\
User 7 & 92.4 & 72.2 & 50.0 & 52.1 & 73.3 \\
User 8 & 92.1 & 51.4 & 100.0 & 50.4 & 73.9 \\
User 9 & 94.0 & 73.5 & 100.0 & 54.5 & 79.5 \\
User 10 & 94.1 & 80.2 & 100.0 & 52.9 & 74.2 \\
Average & 92.2 & 69.2 & 85.0 & & 74.8 \\
\hline
\end{tabular}

achieved a character recognition accuracies of $87.4 \%$, and 92.0\% with elastic matching technique and HMM, respectively. In their study they collected 130 samples of each consonant. The overall stroke recognition accuracy achieved in this work is $93.7 \%$, whereas the average accuracy achieved for identification of characters from recognized strokes is $74.8 \%$ considering all characters and its complex character combinations with nasals and mātrās. As a further work in this direction, one can experiment for achieving higher character recognition rates by building a statistical model for sequence of strokes, which would replace the rule-based approach used in recognition of characters in Gurmukhi script. To improve zone identification algorithm, one can work on dynamically calculating the zone boundaries based on the position of nearby strokes in the character.

\section{Acknowledgements}

We take this opportunity to extend our special thanks to Technology Development for Indian Languages (TDIL), DeitY, MoCIT, Government of India for sponsoring the data collection used in this work.

\section{References}

[1] Tappert C C, Suen C Y and Wakahara 1990 The state of the art in online handwriting recognition. IEEE Trans. Pattern Anal. Mach. Intell. 12(8): 787-808

[2] Jäger S, Liu C-L and Nakagawa M 2003 The state of the art in Japanese online handwriting recognition compared to techniques in western handwriting recognition. Docum. Anal. Recognit. 6(2): 75-88

[3] Liu C-L, Jaeger S and Nakagawa M 2004 Online recognition of Chinese characters: the state-of-the-art. IEEE Trans. Pattern Anal. Mach. Intell. 26(2): 198-213

[4] Yaeger L S, Richard W F I and Pagallo G M 2009 Method and apparatus for acquiring and organizing ink information in penaware computer systems. US Patent 7,564,995. July 21, 2013

[5] Bellegarda E J, Bellegarda J R, Nahamoo D and Nathan K S 1993 A continuous parameter hidden markov model approach to automatic handwriting recognition. Patent EP Patent 0,550,865 July 14

[6] Dutta A and Chaudhury S 1993 Bengali alpha-numeric character recognition using curvature. J. Pattern Recognit. 26 (12): 1757-1770

[7] Garcia-Salicetti S, Doizzi B, Gallinari P, Mellouk A and Fanchon D 1995 A hidden Markov model extension of a neural predictive system for on-line character recognition. In: 
Proceedings of the IEEE Third International Conference on Document Analysis. Canada: 50-53

[8] Hu J, Brown M K and Turin W 1996 HMM based online handwriting recognition. IEEE Trans. Pattern Anal. Mach. Intell. 18(10): 1039-1045

[9] Takahashi K, Yasuda H and Matsumoto T 1997 A fast hmm algorithm for on-line handwritten character recognition. In: Proceedings of IEEE Fourth International Conference on Document Analysis and Recognition. Ulm, Germany: 369-375

[10] Yaeger L, Webb B and Lyon R 1998 Combining neural networks and context-driven search for online, printed handwriting recognition in the newton. AI Mag. 19(1): 73

[11] Plamondon R and Srihari S N 2000 Online and off-line handwriting recognition: a comprehensive survey. IEEE Trans. Pattern Anal. Mach. Intell. 22(1): 63-84

[12] Connell S D, Sinha R M K and Jain A K 2000 Recognition of unconstrained online Devanagari characters. 15th International Conference on Pattern Recognition. Barcelona, Spain: 368-371

[13] Jain A K, Duin R P W and Mao J 2000 Statistical pattern recognition: a review. IEEE Transact. Pattern Anal. Mach. Intell. 22(1): 4-37

[14] Connell S D and Jain A K 2001 Template-based online character recognition. J. Pattern Recognit. 34(1): 1-14

[15] Pechwitz M and Märgner V 2002 Baseline estimation for Arabic handwritten words. In: Proceedings of the 9th International Workshop on Frontiers in Handwriting Recognition. Ontario, Canada: 479-484

[16] Garain U, Chaudhuri B B and Pal T T 2002 Online handwritten Indian script recognition: a human motor function based framework. In: Proceedings of. the 16th International Conference on Pattern Recognition. 3: 164-167

[17] Vijayasenan D, Madhvanath S and Ramakrishnan A G 2004 Principal component analysis for online handwritten character recognition. In: Proceeding of the 17th International Conference on Pattern Recognition. 2: 327-330

[18] Joshi N, Sita G, Ramakrishnan A G, Deepu V and Madhvanath S 2005 Machine recognition of online handwritten Devanagari characters. In: Proceedings of the 8th International Conference on Document Analysis and Recognition. 2: $1156-1160$

[19] Bharath A and Madhvanath S 2007 Hidden Markov models for online handwritten tamil word recognition. In: Proceedings of International Conference on Document Analysis and Recognition. 1: 506-510

[20] Jayaraman A, Chandra Sekhar C and Srinivasa Chakravarthy V 2007 Modular approach to recognition of strokes in Telugu script. In: Proceedings of the 9th International Conference on Document and Recognition. Curitiba, Paraná, Brazil: 501-505

[21] Parui S K, Guin K, Bhattacharya U and Chaudhuri B B 2008 Online handwritten Bangla character recognition using
HMM. Proceedings of the 19th International Conference on Pattern Recognition: 1-4

[22] Sharma A, Kumar R and Sharma R 2008 Online handwritten Gurmukhi character recognition using Elastic Matching. Proceedings of IEEE Congress on Image and Signal Processing. Hainan, China: 391-396

[23] Swethalakshmi H 2008 Online Handwritten Character Recognition of Devanagari and Telugu Characters using Support Vector Machines. MSc Thesis, IIT, Madras, India

[24] Sundaram S and Ramakrishnan A G 2009 An improved online Tamil character recognition engine using post-processing methods. In: Proceedings of the 10th International Conference on Document Analysis and Recognition: 12161220

[25] Sharma A 2009a Online handwritten Gurmukhi character recognition. PhD Thesis, Thapar University, Patiala, India

[26] Prasad M M, Sukumar M and Ramakrishnan A 2009 Divide and conquer technique in online handwritten Kannada character recognition. International Workshop on Multilingual OCR. Catalonia, Spain: 1-7

[27] Sharma A, Kumar R and Sharma R 2009 Rearrangement of recognized strokes in online handwritten Gurmukhi words recognition. In: Proceedings of IEEE International Conference on Document Analysis and Recognition. Barcelona, Spain: 1241-1245

[28] Sharma A, Kumar R and Sharma R 2009b Online pre-processing of handwritten Gurmukhi strokes. Int. J. Mach. Graph. Vis. 18(1): 105-120

[29] Sharma A, Kumar R and Sharma R 2010 HMM-based online handwritten Gurmukhi character recognition. Int. J. Mach. Graph. Vis. 19(4): 439-449

[30] Hajj N and Awad M 2012 Isolated handwriting recognition via multi-stage support vector machines. In: Proceedings of the 6th IEEE International Conference in Intelligent Systems. Bulgaria: 152-157

[31] Sharma R K, Sharma P, Sharma N, Singh H, Verma K, Kumar R and Kumar R 2013 Development of OHWR system for Gurmukhi.VishwaBharat@tdil. 39: 55-66

[32] Kumar R and Sharma R K 2013 An efficient post-processing algorithm for online handwriting Gurmukhi character recognition using set theory. Int. J. Pattern Recognit. Artif. Intell. 27(4): 1353002(1-16)

[33] Kopparapu S K and Lajish V L 2014 A framework for on-line devanagari handwritten character recognition. arXiv preprint arXiv:1410.6909. Accessed 17 September 2016

[34] Guerfali Wacef and Plamondon R 1993 Normalizing and restoring on-line handwriting. J. Pattern Recognit. 26(3): 419-431

[35] Trier $\varnothing$ D, Jain A K and Taxt T 1996 Feature extraction methods for character recognition - a survey. J. Pattern Recognit. 29(4): 641-662 